\title{
Numerical Investigation on the Electrical Performance Optimization of a Tubular Thermoelectric Generator for Waste Heat Recovery
}

\author{
Leisheng Chen ${ }^{1 *}$, Zuyi $\mathrm{Yu}^{1}$, Jingwen Zhang ${ }^{2 *}$, Jaeyoung Lee ${ }^{3}$ \\ ${ }^{1}$ College of Mechanical \& Electrical Engineering, Shaanxi University of Science \& Technology, Xi'an, 710021, China \\ ${ }^{2}$ School of Electronic Science \& Engineering, Xi' an Jiaotong University, China \\ ${ }^{3}$ School of Mechanical \& Control Engineering, Handong Global University, South Korea
}

\begin{abstract}
In the waste heat utilization of automobile exhaust, the tubular thermoelectric generator (TTEG) has structural advantages compared with the flat-plate thermoelectric generator. A kind of TTEG that is composed of $\mathrm{Bi}_{0.5} \mathrm{Sb}_{1.5} \mathrm{Te}_{3}$ and $\mathrm{Ni}$ conical rings alternately attracts researchers' attention, and it generates electrical power based on the transverse thermoelectric effect. However, the electrical performance of such TTEG still needs to be improved for industrial utilization. In this study, the performance of TTEG was optimized through numerical simulation by changing its related structural parameters, including the tilt angle, the thickness of the conical ring, and the relative content of Ni. It is confirmed that the optimal tilt angle with maximum open-circuit voltage $(\mathrm{OCV})$ is $27.3^{\circ}$; on this basis, it is found that a thinner thickness corresponds to a larger OCV; furthermore, when using a conical rings' thickness of $0.75 \mathrm{~mm}$ and increasing the relative content of $\mathrm{Ni}$ in the $\mathrm{Bi}_{0.5} \mathrm{Sb}_{1.5} \mathrm{Te}_{3} / \mathrm{Ni}$ layered pair from $10 \%$ to $90 \%$, the OCV decreases from $198 \mathrm{mV}$ to $105 \mathrm{mV}$ while the power density increases from $413 \mathrm{~W} / \mathrm{m}^{2}$ to $1350 \mathrm{~W} / \mathrm{m}^{2}$. It is believed that these findings can help to develop TTEGs with better electrical performance.
\end{abstract}

\section{Introduction}

Thermoelectric generators (TEG) are devices that convert thermal energy into electricity based on the Seebeck effect. That TEGs do not have moving parts helps to expand the application area, and waste heat recovery utilizing TEGs has attracted much attention from researchers in recent years [1-2], but their thermal-to-electricity conversion efficiency is still relatively low and therefore needs to be improved. Compared to a flat-type TEG, a tubular thermoelectric generator (TTEG) has a structural advantage that enables it to be stuck to a pipe-like heat source or heat sink more closely, then the interfacial thermal resistance is reduced and thermal energy is transferred more efficiently.

Different from custom TTEGs that having potential differences parallel to the temperature gradients [3], a type of TTEG constructed based on transverse thermoelectric effect is also applicable to harvest waste heat. Kanno et al. [4] from Panasonic corporation proposed a TTEG as a heat exchanger, which was composed of $\mathrm{Bi}_{0.5} \mathrm{Sb}_{1.5} \mathrm{Te}_{3}$ and $\mathrm{Ni}$ conical rings alternately, a tilting angle of each layer was formed by looking at the cross-section plane, however, the power density of this kind of TTEG was pretty low. Later on, they optimized the manufacturing process, a method using a melt-spinning technique combined with the spark plasma sintering (SPS) process was applied, which reduced the resistivity and thermal conductivity of the material and therefore increased the power density for a unit heat transfer surface area to 800 $\mathrm{W} / \mathrm{m}^{2}$ and improved the thermoelectric characteristics [5].

To optimize the electrical performance of such TTEGs, the impact of the structural parameters, including the tilt angle of the material layer $\left(15^{\circ}-50^{\circ}\right)$, the thickness of the material layer $(0.5-1.5 \mathrm{~mm})$, and the relative content of $\mathrm{Ni}(10-90 \%)$ on the voltage and power density of a TTEG is numerically investigated in this study. As a result, an optimal tilt angle of $27.3^{\circ}$ corresponding to the maximum open-circuit voltage (OCV) is obtained. It is also found that the thinner the material layer is, the larger the OCV will be; increasing the relative content of $\mathrm{Ni}$ can improve the power density of TTEG. It is believed that the results obtained in this study will provide data references for TEG designers and manufacturers to develop TTEGs with better performances.

\section{Analysis and modelling.}

\subsection{Fundamental principles}

The transverse voltage generated by TTEG is based on transverse thermoelectric effect [6], and it can be described as:

$$
\Delta V=[1 /(2 \mathrm{~d})] \Delta T \Delta S \sin 2 \theta
$$


where $\Delta \mathrm{S}$ is the difference in the Seebeck coefficient between the layer-parallel direction and the layer-perpendicular direction, 1 and $\mathrm{d}$ are the lengths and the thickness of the generator, respectively, $\theta$ is the tilt angle with respect to the layered structure, and $\Delta \mathrm{T}$ is the temperature difference along the radial direction.

The transverse thermoelectric effect uses the nondiagonal elements of the Seebeck coefficient tensor. Due to the artificial anisotropy produced by the arrangement structure of this kind of TTEG and the appropriate value of the tilt angle $\theta$, the non-diagonal element term of the Seebeck coefficient tensor exists. Therefore, TTEG generates the transverse voltage parallel to the axial direction.

\subsection{Numerical model}

The target TTEG is tube-like and has an inner diameter of $10 \mathrm{~mm}$ and an outer diameter of $14 \mathrm{~mm}$. As shown in Fig. 1, it is an inclined layered structure composed of several material layers with a thickness of $1 \mathrm{~mm}$. The tilt angle is set to $30^{\circ}$, the thicknessofthe generator is $2 \mathrm{~mm}$, and the total length is approximately $100 \mathrm{~mm}$. Since COMSOL Multiphysics has already been used to successfully compute the thermal and electrical characteristics of a TEG $[7,8]$, it was also applied to simulate this tubular thermoelectric device and optimize the relevant structural parameters. To simplify the simulation, a 2-D model of the crosssection plane, with a half-length of the tube, was created in this work. Before doing any study with this model, it must be validated.

Fig. 2 shows the geometric structure and highquality meshes of part of the model, which consist of 27365 domain elements and 2087 boundary elements.

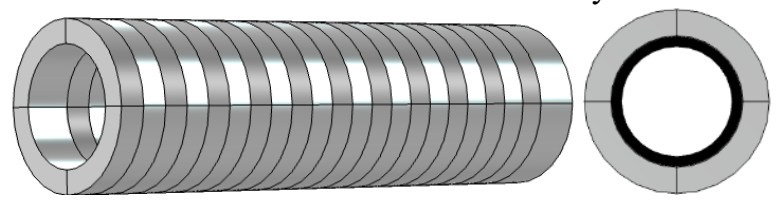

Fig. 1. Side-view (left) and front-view (right) of the TTEG

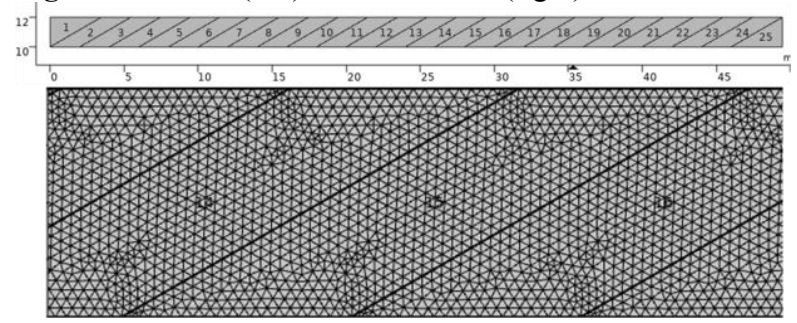

Fig.2. Geometry (Top) and meshes (Bottom) of the 2-D TTEG model

\subsection{Validation of the model}

Firstly, the temperature and flow rate of hot water are $80{ }^{\circ} \mathrm{C}$ and $15 \mathrm{~L} / \mathrm{min}$ respectively, and those of cold water are $10{ }^{\circ} \mathrm{C}$ and $25 \mathrm{~L} / \mathrm{min}$ respectively. The distribution of temperature and $\mathrm{OCV}$ in the layered structure is obtained by two-dimensional finite element analysis as shown in Figure 3. Observing the simulation results of the temperature distribution, it is found that the isotherms are equidistant linearly distributed in the $\mathrm{Ni}$ layer while twisted in the $\mathrm{Bi}_{0.5} \mathrm{Sb}_{1.5} \mathrm{Te}_{3}$ layer. Furthermore, the open circuit voltage develops along the axial direction due to the transverse thermoelectric effect.

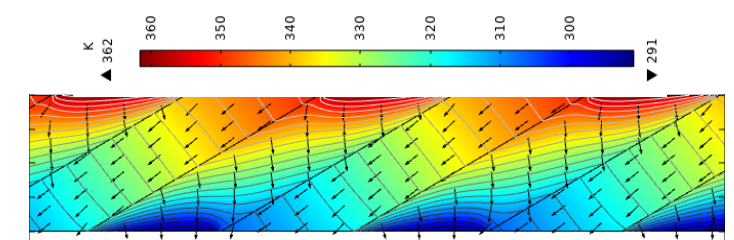

(a)

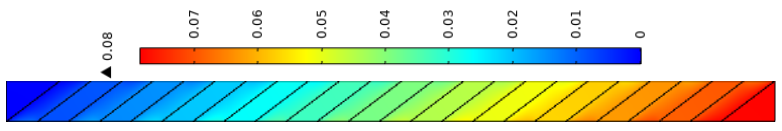

(b)

Fig. 3. (a) Temperature profile and heat flux flow direction;

(b) Potential distribution in the TTEG

Keeping boundary conditions of the cold side constant, varying the hot side temperature or flow rate, the OCV is observed and the results plotted in Figures $4 \mathrm{a}$ and $\mathrm{b}$. The circles represent the experimental results, and the solid line and double dots line are the numerical results acquired in the literature [4] and this study.

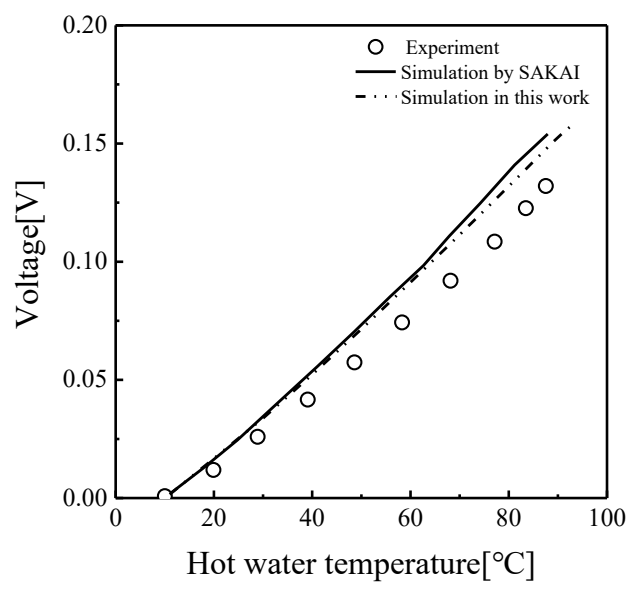

(a)

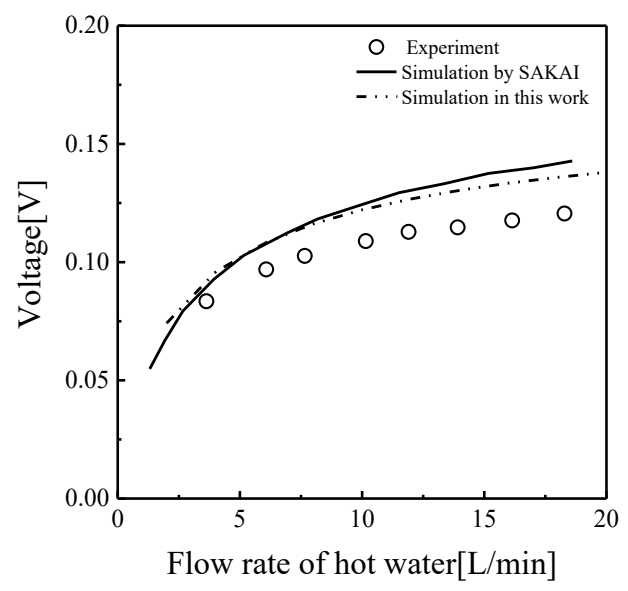

(b)

Fig. 4. Open circuit voltages under different (a) hot-side temperatures and (b) flow rates of hot-side fluid 
It is found that, as shown in Fig. 4a, when the hot water temperature rises from $10{ }^{\circ} \mathrm{C}$ to about $90{ }^{\circ} \mathrm{C}$, the OCV obtained by the present model shows a better linear trend and is closer to the experimental values than the numerical model proposed in the literature, especially when the hot water temperature is in the range of $60{ }^{\circ} \mathrm{C}$ to $90{ }^{\circ} \mathrm{C}$. Moreover, in Fig. $4 \mathrm{~b}$, when the flow rate of hot water rises from $2 \mathrm{~L} / \mathrm{min}$ to 20 $\mathrm{L} / \mathrm{min}$, the OCV of the model applied in this paper is closer to the experimental value and the saturation trend is more significant at a high hot-water flow rate.

These results are in good agreement with those obtained by Kanno et al.[4], therefore, the model established in this paper is reliable and practical and can be used to observe more characteristics of TTEG.

\section{Results and discussion}

The validated model was applied to optimize the related structural parameters of TTEG, including the tilt angle, the layer thickness, and $\mathrm{Ni}$ content. These parameters were changed under the conditions of hot water temperature and flow rate of $93{ }^{\circ} \mathrm{C}$ and 15 $\mathrm{L} / \mathrm{min}$; cold water temperature and flow rate of $10{ }^{\circ} \mathrm{C}$ and $25 \mathrm{~L} / \mathrm{min}$.

\subsection{Optimization of the tilt angle}

Firstly, the tilt angle of the layered structure is changed when the thickness of the material layer is kept at 1 $\mathrm{mm}$. Fig. 5a shows the open circuit voltages corresponding to different tilt angles, and a parabolic curve is acquired as the tilt angle increases from $15^{\circ}$ to $50^{\circ}$. After fitting the simulation results, the function of $\mathrm{OCV}$ to tilt angle is obtained as $y=0.079+0.0059 x-0.000108 x^{2}$, and the optimal tilt angle with maximum $\mathrm{OCV}$ is $27.3^{\circ}$. The variation of the power density with different tilt angles is shown in Fig. 5b, unlike the voltage, the power density keeps increasing with the decrease of tilt angle. The maximum power density is around $1000 \mathrm{~W} / \mathrm{m}^{2}$ when the tilt angle comes to $15^{\circ}$, and it will become higher as the tilt angle gets smaller. The power density of around $500 \mathrm{~W} / \mathrm{m}^{2}$ is obtained under the optimal tilt angle

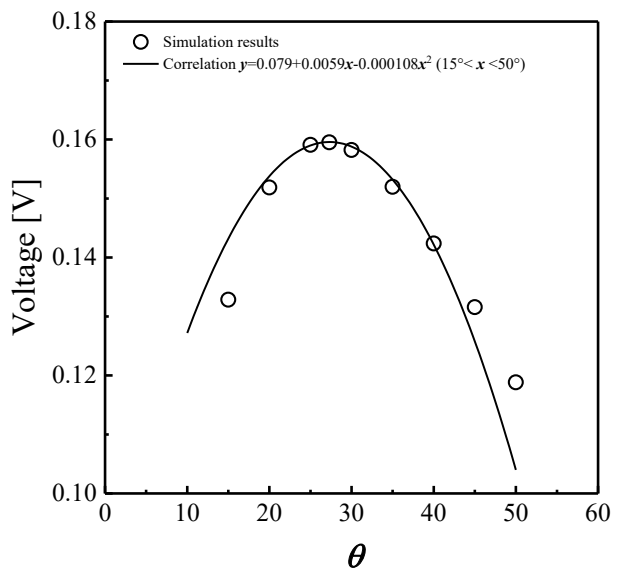

(a)

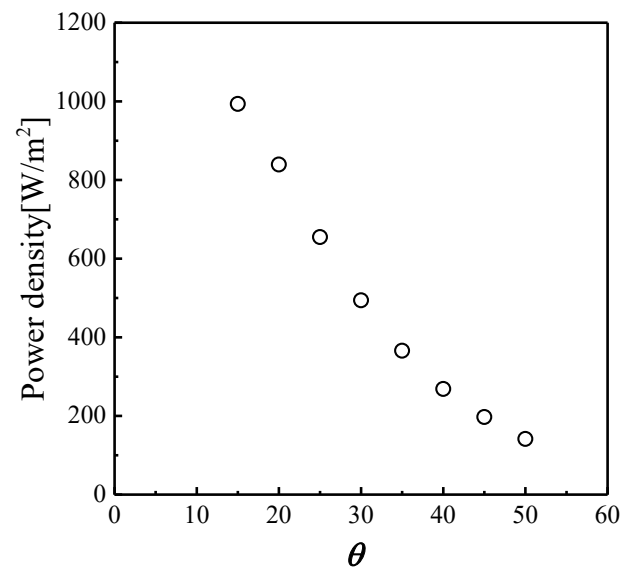

(b)

Fig.5. Variation of (a) voltage and (b) power density with the tilt angle

\subsection{Effect of the layer thickness}

The impact of the thickness of the material layer on the OCV is studied under the optimal tilt angle, and the results are shown in Fig. 6. It can be seen that the OCV decreases as the layer thickness increases from $0.5 \mathrm{~mm}$ to $1.5 \mathrm{~mm}$. However, it does not necessarily mean that the thickness must be thinner to gain better performance of TTEG, because the electrical resistivity of each material also varies along with the thickness and it might weaken the output power. Therefore, it is better to choose a thickness by balancing the resistance and OCV out. In this study, a more practical material layer thickness of $0.75 \mathrm{~mm}$ is selected as the basis for further optimization, and the total thickness of $\mathrm{Bi}_{0.5} \mathrm{Sb}_{1.5} \mathrm{Te}_{3} / \mathrm{Ni}$ layered pair is $1.5 \mathrm{~mm}$.

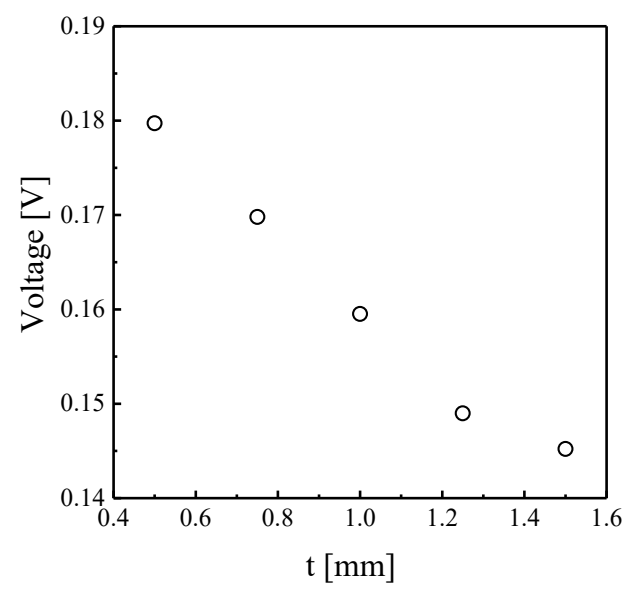

Fig.6. Variation of open circuit voltage with the layer thickness

\subsection{Effect of the relative content of $\mathrm{Ni}$}

While keeping the total thickness of $\mathrm{Bi}_{0.5} \mathrm{Sb}_{1.5} \mathrm{Te}_{3} / \mathrm{Ni}$ layered pair unchanged, change the relative content of $\mathrm{Ni}$ in the $\mathrm{Bi}_{0.5} \mathrm{Sb}_{1.5} \mathrm{Te}_{3} / \mathrm{Ni}$ layered pair by changing the percentage of Ni layer thickness to the total thickness 
of the layered pair, increasing the relative content of $\mathrm{Ni}$ in the $\mathrm{Bi}_{0.5} \mathrm{Sb}_{1.5} \mathrm{Te}_{3} / \mathrm{Ni}$ layered pair from $10 \%$ to 90 $\%$, the OCV and power density have different trends. The open circuit voltage and the power density values corresponding to the different relative content of $\mathrm{Ni}$ obtained by simulation are respectively in Figures $7 \mathrm{a}$ and $b$. The OCV decreases from $198 \mathrm{mV}$ to $105 \mathrm{mV}$ (Fig. 7a) while the power density increases from 413 $\mathrm{W} / \mathrm{m}^{2}$ to $1350 \mathrm{~W} / \mathrm{m}^{2}$ (Fig. $7 \mathrm{~b}$ ). If high power density is preferred, the relative content of $\mathrm{Ni}$ in the $\mathrm{Bi}_{0.5} \mathrm{Sb}_{1.5} \mathrm{Te}_{3} / \mathrm{Ni}$ layered pair can be appropriately increased. At this time, the consumption of the thermoelectric material $\mathrm{Bi}_{0.5} \mathrm{Sb}_{1.5} \mathrm{Te}_{3}$ is reduced, so the manufacturing cost is reduced and the economy is improved.

It also can be found that when the relative content of $\mathrm{Ni}$ is in the range of $10 \%$ to $30 \%$ and $50 \%$ to 90 $\%$, the open circuit voltage has a significant downward trend; when the relative content of $\mathrm{Ni}$ is in the range of $30 \%$ to $50 \%$, the open circuit voltage changes slightly. Besides, the increase rate of power density in the range of $30 \%$ to $90 \% \mathrm{Ni}$ relative content is significantly higher than that in the range of $10 \%$ to 30 $\%$.

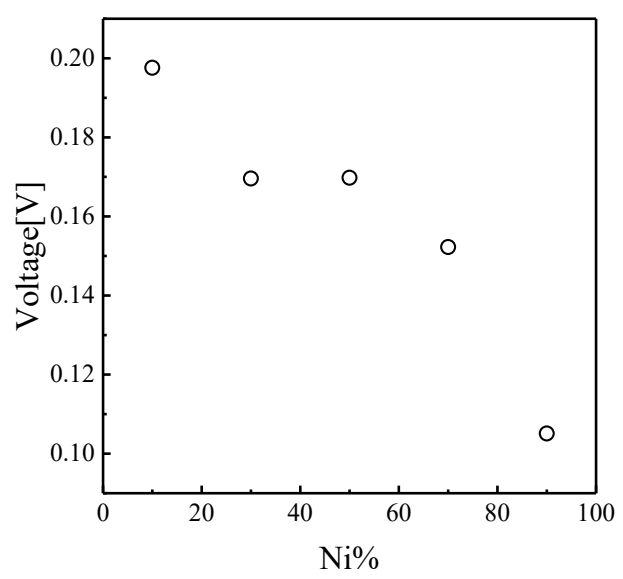

(a)

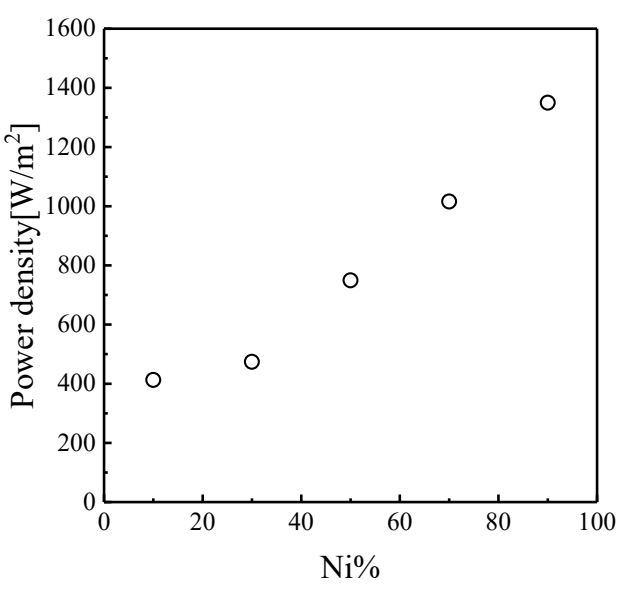

(b)

Fig.7. Variation of (a) open circuit voltage and (b) power density with the relative content of $\mathrm{Ni}$

\subsection{Output power of the optimized TTEG}

If the resistance of the external resistor connected to TTEG is changed, the load voltage on the external resistor will change. Fig. 8 is the output power value corresponding to the load voltage of different external resistors obtained by simulation. At this time, the optimal angle of $27.3^{\circ}$ is used and the total thickness of $\mathrm{Bi}_{0.5} \mathrm{Sb}_{1.5} \mathrm{Te}_{3} / \mathrm{Ni}$ layered pair is taken as $1.5 \mathrm{~mm}$. When the resistance of the external resistor and the internal resistance of TTEG are equal, the load voltage of the external resistance is $0.085 \mathrm{~V}$ and the maximum output power is $2.35 \mathrm{~W}$.

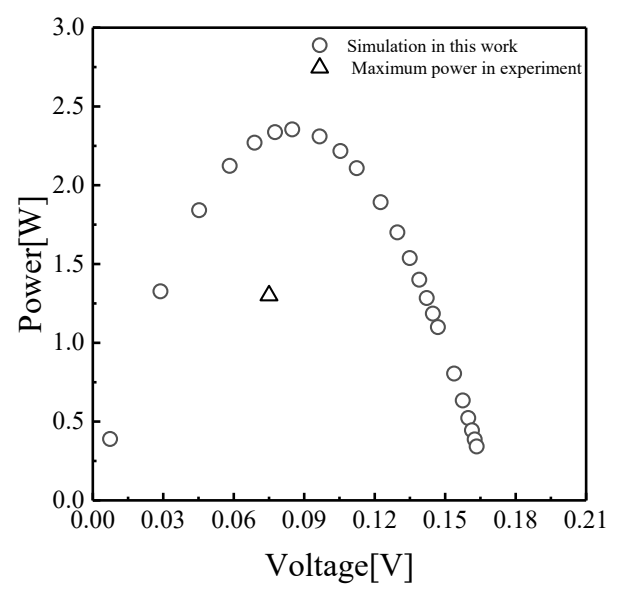

Fig.8. The optimized performance of TTEG

The triangle in Fig. 8 represents the maximum output power, $1.3 \mathrm{~W}$, obtained by the TTEG without performance optimization in reference [4]. Through optimization, the maximum output power of TTEG has been increased by $81 \%$, which shows that the optimization in this study has significant effects and application significance on TTEG.

\section{Conclusions}

In this study, an optimization on electrical performances of TTEG is carried out using COMSOL to improve the open-circuit voltage or output power. The temperature distribution and electric potential distribution in the used TTEG model were obtained through two-dimensional finite element analysis and compared with the previous models.

First, the influence of the tilt angle on the thermoelectric characteristics of TTEG is studied. Then, the open-circuit voltage generated by the model with different material layer thicknesses was obtained by simulation. Furthermore, when the TTEG adopts a tilt angle of $27.3^{\circ}$ and a material layer pair of $1.5 \mathrm{~mm}$ thick, the impact of the relative content of $\mathrm{Ni}$ on the thermoelectric characteristics is also studied. Finally, the function of load voltage and output power is obtained by changing the external resistance of the optimized TTEG, and compared with the maximum output power generated by the TTEG without performance optimization. According to the results, the following conclusions are drawn: 
1). When only the tilt angle is changed, the opencircuit voltage presents a parabolic distribution within a certain tilt angle range, and the optimal tilt angle is determined to be $27.3^{\circ}$; when the tilt angle is from $15^{\circ}$ to $50^{\circ}$, the power density generated by TTEG decreases with the increase of tilt angle.

2 ). When the thickness of the material layer is changed based on the optimal tilt angle, it is found that the open-circuit voltage increases as the thickness of the material layer decreases. It's found that the opencircuit voltage of $0.75 \mathrm{~mm}$ thick TTEG is $6 \%$ higher than that of $1 \mathrm{~mm}$ thick TTEG.

3). In TTEG with a tilt angle of $27.3^{\circ}$ and a material layer pair of $1.5 \mathrm{~mm}$ thick, when the relative content of $\mathrm{Ni}$ rises from $10 \%$ to $90 \%$, the open-circuit voltage gradually decreases, while the power density gradually increases.

4). After adopting the optimal tilt angle and thinner material layer thickness, the maximum output power of TTEG is increased by $81 \%$ compared with TTEG without performance optimization.

These results are useful and helpful for TEG designers to develop TTEGs with better electrical performances, which will enhance the application of TTEGs in waste heat recovery.

This work was supported by start-up young researchers fund (2019-BJ07) granted by Shaanxi University of Science \&Technology and funded by China Postdoctoral Science Foundation (No.2021M691999).

\section{References}

1. A. Kobayashi, R. Konagaya, S. Tanaka, M. Takashiri, Sensor Actuat A-Phys. 313 :112199 (2020)

2. L. Chen, J. Lee, Energy Conver. Manage. 119 :pp 75-80 (2016)

3. X. Tian, S. Asaadi, H. Moria, A. Kaood, S. Pourhedayat, K. Jermsittiparsert, Energy. 208 :118428 (2020)

4. T.Kanno, A. Sakai, K. Takahashi, A. Omote, H. Adachi, Y. Yamada, Appl. Phys. Lett. 101 :011906 (2012)

5. A. Sakai, T. Kanno, K. Takahashi, H. Tamaki, H. Adachi, Y. Yamada, J. Electronic Materials 42: pp1612-1616 (2013)

6. K. Takahashi, A. Sakai, T. Kanno, H. Adachi, Appl. Phys. Lett. 95, 051913 (2009)

7. L. Chen, J. Lee, Appl. Energy. 150 :pp 138 149(2015)

8. H. Luo, L. Chen, Numerical study on optimizing the geometry of segmented asymmetrical generator under pulse heat power. The 5th International Conference on New Energy and Future Energy System, 3-6 Nov. 2020, Xi'an, China, (2020) 